\title{
Innovation of Corporate Governance: A Discussion based on Blockchain Technology
}

\author{
Ziwei Wang \\ Sydney Institute of Language and Commerce, Shanghai University, Shanghai 200444, China \\ wangziwei1218@163.com
}

\begin{abstract}
Blockchain, as a newly-born application of computer technology, has unique advantages in data storage, point-to-point transmission, consensus mechanism and encryption algorithm, which could lead to innovation of corporate governance. The application of blockchain technology in corporate governance has attracted more and more attention as it provides new approaches to improve corporate innovation ability as well as operation efficiency. This paper will discuss three types of applications of blockchain technology in corporate governance, including operating based on DAO, creating employee performance appraisal and incentive mechanism and cooperating among Multi-departmental employees. There are three aspects that will be included in the discussion of each application, namely mechanism, preconditions and benefits, so as to show the potentials of blockchain technology comprehensively. Furthermore, this paper will provide detailed design of corporate governance model, including the structure of Blockchain-based platforms, design of operating system and design of appraisal and incentive system, which offers a guideline to corporations that intends to adopt blockchain technology in their corporate governance.
\end{abstract}

Keywords: Blockchain; Corporate Governance; DAO; Smart Contract; Token.

\section{Introduction}

Blockchain technology has been recognized as one of the most revolutionary technologies in the era of digitalization. The use of blockchain technology could create and increase socio-economic welfare [1] in the industries of finance, game, medicine and Internet. The most familiar application of blockchain is corporate finance, including Initiate Coin Offering (ICO) and supply chain finance. Moreover, blockchain has potentials to change the ways that traditional corporations work and to deal with problems that exist in the traditional corporate governance. Blockchain technology could decentralize corporate structure and create a consensus-based operating system called Decentralized Autonomous Organization (DAO).[2]DAO could improve information sharing, performance evaluation and cross-departmental cooperation. This would ameliorate innovation ability and operation efficiency of corporations, and thus attracting business leaders, the decision makers of corporations, to make trials on the applications of blockchain technology and to explore the innovative models of corporate governance.

Blockchain is a newly-born application mode of computer technology, having advantages in distributed data storage, point-to-point transmission, consensus mechanism and encryption algorithm.[3]It has five characteristics that jointly form its value: 1. Distribution, 2. Encryption, 3. Tampering proof, 4. Tokenization and 5. Decentralization. Although it is difficult to include all of the characteristics in the current application of blockchain technology, there is a growing trend to explore the great potentials of blockchain in corporations. David Furlonger \& Christophe Uzureau (2020) have divided the development of blockchain into three stages, in which the blockchain achieves limited (2012-2023), part (2023-2025) and full (2025-) potentials in transferring 'traditional organizations' into 'blockchain organizations'.[4] Due to the influence of COVID-19, there are now an increasing number of corporations adopting blockchain technology in their daily operations, which certificates the advantages and potentials of blockchain technology in corporate governance.

This paper is organized as follows. Section 2 will discuss about applying blockchain to corporate governance, including operating based on DAO, creating employee performance appraisal and incentive mechanism and cooperating among Multi-departmental employees. Section 3 will introduce 
the design of corporate governance model, focusing on Blockchain-based platforms, operating system and appraisal and incentive system. Finally, section 4 will conclude the whole study.

\section{Discussion about Applying Blockchain to Corporate Governance}

Since blockchain technology has aforementioned five characteristics, it could alter the methods of corporate governance and improve the efficiency of corporate operation. In relation to the blockchain technology, there are three types of applications to be discussed: operating based on DAO, creating employee performance appraisal and incentive mechanism and cooperating among Multidepartmental employees.

\subsection{Operating based on DAO}

\subsubsection{Mechanism}

Blockchain technology can help companies operate based on DAO due to the characteristics of distribution and decentralization. Companies that adopt blockchain technology can establish decentralized Blockchain-based platforms in order to realize collective co-governance and improve the efficiency of corporate governance. These platforms are built using smart contracts, which are created by complex algorithms. Smart contracts can automatically verify the conditions that are included in the algorithms without any human intervention. When certain conditions are met, Blockchain-based platforms will operate and make decisions automatically based on smart contracts, and thus formulating a consensus-based operating system 'DAO'. DAO can deal with both simple and complex matters, allowing both individuals and departments to deal with problems rapidly and accurately. Moreover, employees and leaders are able to make rules and standards through DAO to participate in the corporate governance. Token, as the proof of work (Pow) and the proof of stake (Pos), allows employees to turn their works and abilities into influence when making rules and standards. Since blockchain technology has the characteristic of distribution, it would be difficult for a single person or department to tamper DAO. Hence, Blockchain-based platforms could process and transmit data according to smart contracts accurately.

\subsubsection{Preconditions}

There are two important preconditions that should be met if a corporation intends to accept blockchain technology and operate based on DAO.

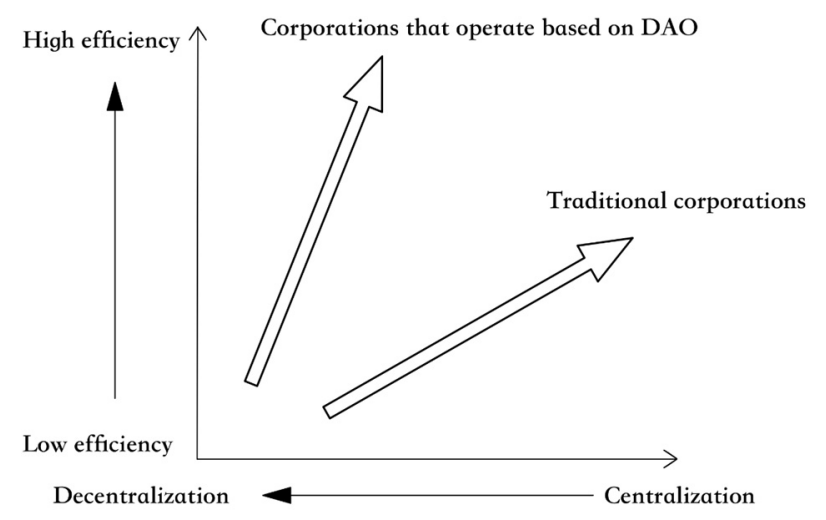

Fig 1. Relationship between efficiency and decentralization

Firstly, companies that intend to operate based on DAO should be willing to accept blockchain technology. This requires leaders to be active when adopting blockchain technology instead of being forced to accept it in corporate governance. Moreover, since operating based on DAO requires participation of every employee, leaders should be open to new things and be ready to authorization. Those who are forced to accept blockchain technology, called 'Fear of missing' and 'Trojan horses', 
may ignore the advantages of blockchain such as distribution and decentralization, and thus failing to save costs and operate effectively based on DAO.[5] This provides a simple method to evaluate whether the precondition is met, which is to evaluate the degree of adaptation to decentralization (Fig. 1). Generally, operation efficiency would be positively correlated with the degree of decentralization.

Another precondition is the operating environment of corporations. As is mentioned before, DAO is constructed by smart contracts, which are algorithms in nature. In other words, DAO is not capable of dealing with irregular things in corporate operations. Although DAO could cope with both simple and complex matters, individuals and departments still need to deal with unusual matters or turn matters into algorithms. Therefore, it is important for corporations to focus on those highly standardized, coded or automated processes and then expanding the functions of DAO by turning irregular matters into algorithms.

\subsubsection{Benefits}

Corporations that operate based on DAO would benefit from three aspects.

Firstly, corporate capability and efficiency of dealing with matters could be improved. Due to the distribution and decentralization of blockchain, corporations that operate based on DAO would have more decentralized structures. Hence, information could be transmitted directly and DAO could automatically deal with matters that are included in the smart contracts, which could improve the capability and efficiency of operations.[6]

Then, the cost of corporations could be saved during operations, including both time cost and labor cost. Since DAO could automatically deal with matters accurately, employees would be able to focus on more essential and irregular things, and thus saving time cost for corporation. Moreover, the influence of human factors in corporate operations could be alleviated as DAO could operate automatically, and therefore saving labor cost for human intervention. [7] Although creating DAO would incur initial expenses, the cost-saving effects of DAO during operations would far outweigh its costs.

Finally, DAO could improve the innovation ability of corporations. [8] Blockchain technology could build decentralized Blockchain-based platforms based on a consensus-based system to aid corporate operations. It requires trust between employees and leaders to maintain operations based on DAO. Since trust would help employees give full play to their subjective initiative and be willing to provide innovative ideas for the company, it would improve corporate innovation ability.[9]

\subsection{Creating Employee Performance Appraisal and Incentive Mechanism}

\subsubsection{Mechanism}

Blockchain technology is capable of creating employee performance appraisal and incentive mechanism in corporate governance. Unlike DAO, this mechanism is created mainly based on the characteristics of tokenization. Since Blockchain-based platforms can record data and process matters automatically, it enables real time dynamic appraisal of employee performance through smart contracts. When employees accomplish certain tasks, Blockchain-based platforms will evaluate employee performance based on rules and standards of DAO and send tokens as rewards. Moreover, leaders can reward employees through platforms according to the results of appraisal, which may become an effective incentive mechanism of corporate governance. These rewards, known as tokens, are similar to virtual points and are difficult to be tampered. As for the types of token, Proof of work (Pow) stands for number and quality of works, while Proof of stake (Pos) stands for contributions to corporation.[10] Employees can exchange Pos for money or other types of rewards through platforms. Moreover, the number of Pos stands for the influence of employees and plays an important role in making rules and standards in DAO. Therefore, this mechanism could ensure that employee performance being appraised accurately and timely, while the incentive mechanism could be fair and acceptable to all the employees. 


\subsubsection{Preconditions}

There are two preconditions that correlate with creating employee performance appraisal and incentive mechanism in corporate governance.

Firstly, the acceptance of rules and appraising standards among employees and leaders is essential to this mechanism. Since smart contracts are the basis of the consensus-based platforms, it is necessary to reach an agreement between employees and leaders concerning rules and standards of appraisal and incentive so that this mechanism could be efficient and fair for corporations. Moreover, this mechanism requires corporations to decentralize their governance and to realize collective cogovernance.

Then, the intensity of competition in the corporation will affect the efficiency of this mechanism, making it become another precondition (Fig. 2). When corporations face fierce competitions in daily operations, this mechanism would be useful in both appraisal and incentive as employees could gain fair and accurate assessments and rewards for their hard works, which may encourage them to work and compete even harder for better results. Due to DAO, most employees would be ready to accept this mechanism, and thus resulting in the amelioration of efficiency.[11]

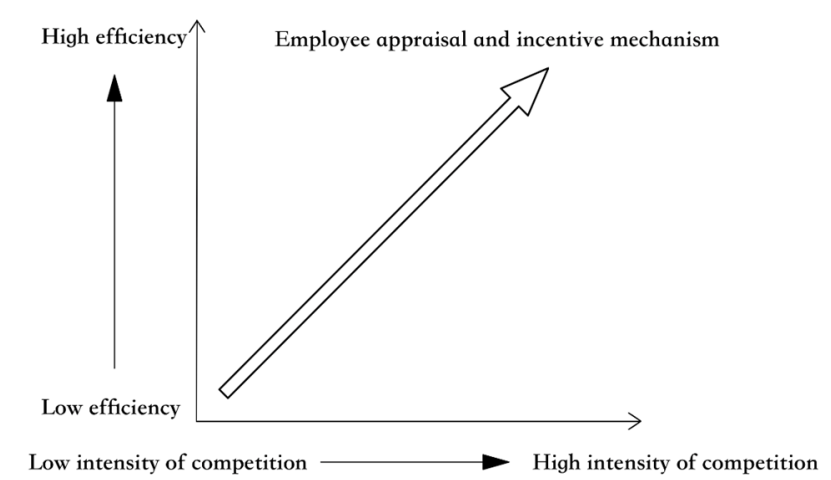

Fig 2. Relationship between efficiency and intensity of competition

\subsubsection{Benefits}

Benefits of this mechanism would be classified into two parts.

Firstly, employees would make greater contributions to the corporations. Employees should constantly make contributions to their corporations to gain better assessments and rewards under this mechanism. Otherwise, their influence and roles would be weakened as Blockchain-based platforms have dynamic appraisal of their contributions. On the other hand, employees would become more creative than before in their works, which would benefit both corporations and themselves.

Moreover, employees would make fewer complaints about their corporations. Since creating employee performance appraisal and incentive mechanism would require participation of both employees and leaders, there would be a higher acceptable level during corporate operations. Besides, this mechanism would be fair to each member of the corporations as tampering smart contracts in the Blockchain-based platforms would be difficult. [12] This would result in lower employee turnover rates and greater corporate efficiency.

\subsection{Cooperating among Multi-departmental Employees}

\subsubsection{Mechanism}

Blockchain technology can realize cooperation among Multi-departmental employees in corporate governance based on the characteristics of decentralization and tampering proof. Blockchain-based platforms play important roles in this mechanism as they could save and transmit data timely and accurately. When employees have accomplished their works, smart contracts will automatically save and transit related data to the Blockchain-based platforms. Every information will be encrypted and timestamped to prevent tampering. Every participant of these platforms is able to screen and search for the useful information that are saved in these platforms. Due to DAO, employees and leaders have 
equal access to all of the information in these platforms, which could alleviate information asymmetry. Moreover, the use of this mechanism will eliminate the boundary between different departments and decentralize the departments as employees can exchange information with those from other departments directly, and therefore realizing the cooperation among Multi-departmental employees.

\subsubsection{Preconditions}

There are two preconditions that relate to the cooperation among Multi-departmental employees.

Firstly, the acceptance of altering functions and styles of leadership, which may become a challenge for many traditional centralized corporations. [13] Since boundary between different departments will be eliminated through this mechanism, leaders should change their functions and styles in corporate governance so as to adapt themselves to the mechanism (Fig. 3). Leaders should be more active to participate in corporate governance and have a kind of honeycomb thinking. They should not have a fixed role and should give frequent feedback to their employees. Moreover, they should focus on helping, encouraging their employees and should link resources and provide abilities if necessary.

\begin{tabular}{|c|c|c|}
\hline & Traditional leadership & Blockchain-based leadership \\
\hline Participation & Passive & Active \\
\hline Thinking method & Linear thinking & Honeycomb thinking \\
\hline Role & Fixed & Mobile \\
\hline Work focus & Monitoring & Encouraging \\
\hline Influence & Tutoring & Linking resources \\
\hline Management style & Allocating resources & . \\
\hline
\end{tabular}

Fig 3. Contrasts between traditional leadership and Blockchain-based leadership

On the other hand, this mechanism requires rapid adaptation to the environment. Cooperating among Multi-departmental employees would lead to a more complex environment in corporate governance, with information being updated rapidly and relationships between employees being changed frequently. Hence, employees and leaders should adapt themselves to the environment as soon as possible to deal with issues properly.

\subsubsection{Benefits}

Cooperating among Multi-departmental employees would bring two types of benefits to the corporate governance.

First and foremost, information asymmetry could be alleviated. Since information could be transmitted through Blockchain-based platforms, employees would be able to gain information directly and timely. [14] Moreover, information would be more accurate as it will be encrypted and timestamped in this mechanism. As a result, the corporate ability of dealing with different issues could be improved through this mechanism.

On the other hand, employee would be able to improve their abilities in working and cooperating. This mechanism emphasizes the participation and cooperation of employees, accompanied with aid of leaders. Employees could improve their working cooperating ability and be encouraged by leaders to work harder, which would ameliorate the efficiency of corporate governance.

\section{Design of Corporate Governance Model based on Blockchain Technology}

Apart from understanding the changes blockchain technology could bring to corporate governance, it is essential to learn how to apply blockchain technology in real environment. This section will 
provide further information relating to the design of corporate governance model so as to give readers a clear guideline when applying blockchain technology to corporate governance.

\subsection{Structure of Blockchain-based Platforms}

Blockchain-based platforms are not only the basis of DAO, but also the key to applying blockchain technology to corporate governance. As is mentioned in section 2, Blockchain-based platforms have several functions: 1. Saving data, 2. Transmitting data, 3. Screening data, 4. Processing matters, 5. Making rules and standards, 6. Encryption and 7. Issuing tokens. The structure of Blockchain-based platforms is shown as follows:

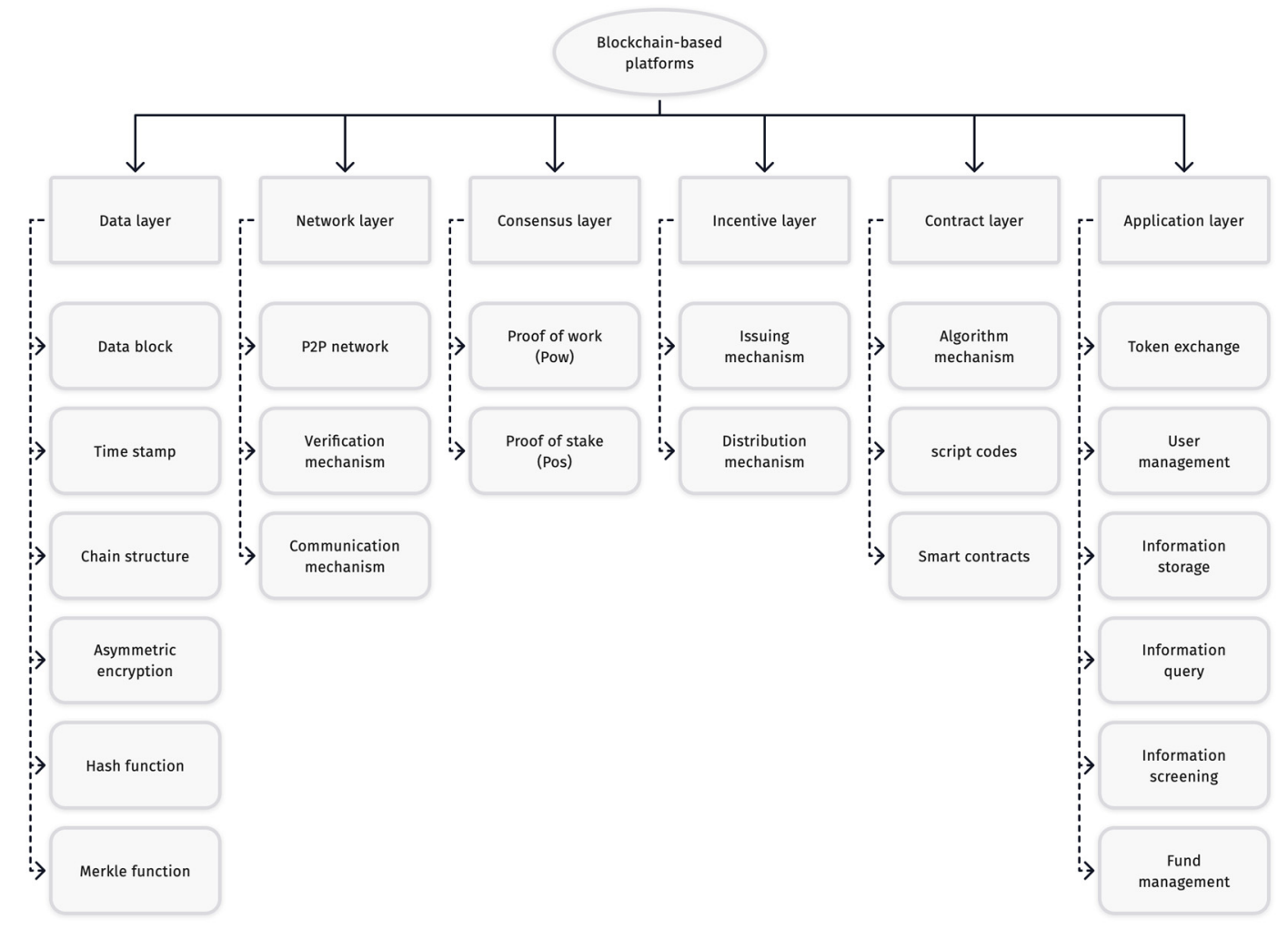

Fig 4. Structure of Blockchain-based platforms

In Fig. 4, it is clear that Blockchain-based platforms have six layers, namely data layer, network layer, consensus layer, incentive layer, contract layer and application layer.

Data layer contains data block, time stamp, chain structure, asymmetric encryption, Hash function and Merkle function. [15] as for corporate governance, every employee and leader will enter the Blockchain-based platforms with a unique address, which is created by Hash function. Blockchainbased platforms will encrypt all the personal information as well as working documents using time stamp, asymmetric encryption, Hash function and Merkle function in order to prevent tampering. Information will be saved in data blocks and be transmitted according to chain structure such as private chain, alliance chain and public chain. Data layer is the core structure of Blockchain-based platforms.

Network layer includes P2P network, verification mechanism and communication mechanism, which realizes information exchange between blockchain network nodes. [16] In corporate governance, every employee and leader, acting as a node, will constitute a P2P network to exchange information and documents with others in Blockchain-based platforms. Blockchain-based platforms will verify the identity of participant according to the verification mechanism and license. After exchange, each node will actively send and automatically verify information based on communication mechanism to ensure its accuracy and timeliness. 
Consensus layer contains proof of work (Pow) and proof of stake (Pos). As for corporate governance, consensus layer is the basis of DAO and employee performance appraisal and incentive mechanism. Pow can be used to reward employees for their hard works, while Pos can be used to make rules and standards in DAO. The existence of Pow and Pos leads to formulation of collective co-governance and improve the efficiency of corporate governance.

Incentive layer includes issuing mechanism and distribution mechanism. In corporate governance, issuing mechanism and distribution mechanism serves for making and distributing tokens such as proof of work (Pow) and proof of stake (Pos). Blockchain-based platforms can automatically send Pow and Pos to nodes according to smart contracts, which ensures accuracy and fairness.[17]

Contract layer contains algorithm mechanism, script codes and smart contract. [18] Contract layer plays an important role in ensuring the operation of Blockchain-based platforms. Algorithm mechanism and script codes jointly constitute the platforms, while smart contract ensures the normal operations of Blockchain-based platforms.

Application layer includes token exchange, user management, information storage, information query, information screening and fund management. In corporate governance, application layer stands for the various functions of Blockchain-based platform. Every participant could have access to these functions, which leads to the realization of operating based on DAO, creating employee performance appraisal and incentive mechanism and cooperating among Multi-departmental employees.

\subsection{Design of Operating System}

Operating system is the core of corporate governance, including operating based on DAO and cooperating among Multi-departmental employees. Although operating system may differ from corporation to corporation due to their size, industry and operation status, the logic of designing operating system remains unchanged. The design of operating system is shown in Fig. 5:

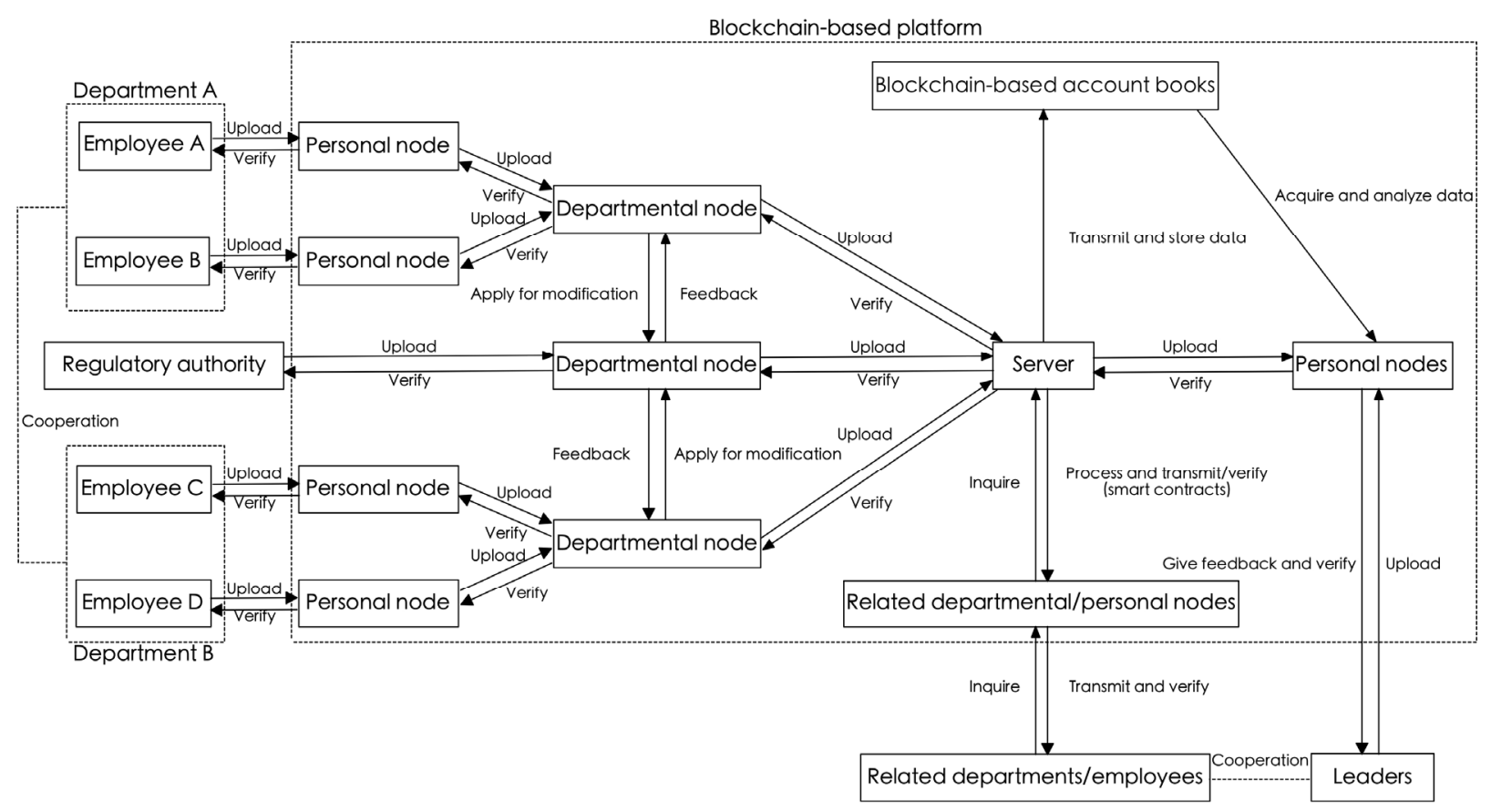

Fig 5. Design of operating system

There are several steps that should follow in the operating system in order to transmit and process data and documents. Firstly, employees should upload data and documents to personal nodes, which are usually known as computers. The personal nodes will automatically verify the completeness of data and documents as well as the identity of employees, and then uploading data and documents to 
departmental nodes. Departmental nodes will repeat similar steps and upload all of the data and documents to the server, acting as the bridges between personal nodes and server. Server, the core of Blockchain-based platform, will verify the completeness and identity again, and processing and transmitting these data and documents automatically based on smart contracts. As is mentioned before, smart contracts are the basis of DAO, which are the consensus of both employees and leaders. Finally, related departments or employees can inquire and screen data and documents and related departmental or personal nodes will transmit these data and documents to related departments or person. This allows employees to cooperate with employees from other departments rapidly and directly as there is no intervention of others during the course. If leaders intend to transmit and process data and documents, the steps are similar to those of employees. Owing to the characteristic of encryption, data and documents will be encrypted using Hash function and time stamp during transmission and processing to prevent tampering. Besides, all the data and documents will be saved in distributed Blockchain-based account book, which exist in every node, and therefore ensuring the safety and accuracy of data and documents.

Moreover, this operating system provides solution to errors that happen during transmission. If regulatory authority find errors, it can upload related documents to its departmental nodes and give feedback to related employees or departments through their personal nodes or departmental nodes. However, if employees or departments find errors in advance, they can apply for modification through personal and departmental nodes, and then uploading their modified data and documents.

On the other hand, leaders can gain and analyze data and make improvements if necessary. Leaders can acquire data and documents directly from Blockchain-based account books after verifying their identities. This could help leaders find issues that may exist in the current operating system, but also improve cooperation between leaders and employees.

In general, the design of operating system takes advantages of blockchain's characteristics like encryption and distribution to realize operating based on DAO. Moreover, since data and documents could be transmitted and processed accurately and rapidly, corporations would be decentralized and cooperating among Multi-departmental employees would become more efficient.

\subsection{Design of Appraisal and Incentive System}

The design of appraisal and incentive system focuses on the use of tokens to evaluate the employees' works and to ameliorate innovation and efficiency of employees. This section will introduce token issuing and token exchange respectively.

\subsubsection{Token Issuing}

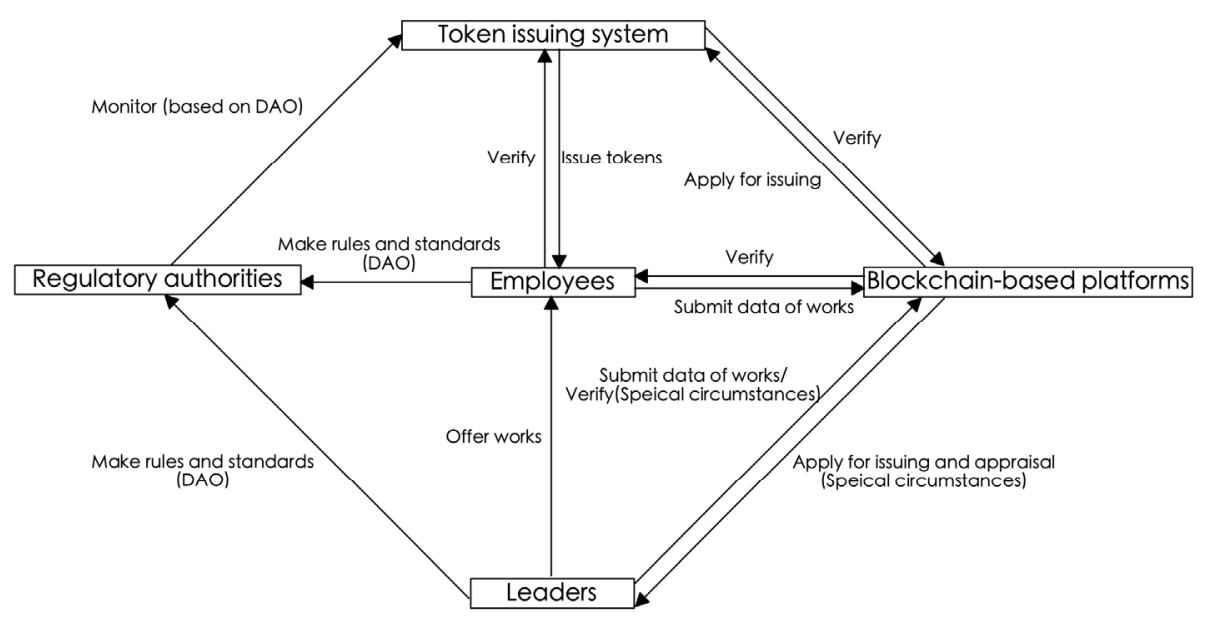

Fig 6. Token issuing

Fig. 6 shows the mechanism of token issuing in the appraisal and incentive system, which can be used to assess and reward employees for their works. When employees accomplish the tasks, they 
need to submit data or documents of works to the Blockchain-based platforms. Blockchain-based platforms will verify the identities as well as evaluate the quality and quantity of tasks based on smart contracts. Smart contracts contain both rules and standards that are made based on DAO so as to ensure accuracy and fairness of evaluation. Blockchain-based platforms will then apply to token issuing system for token issuing. token issuing system will verify the identity of employees and the number of tokens, and then issuing tokens like Pow and Pos to the digital wallets of employees. Employees should verify the numbers of tokens received and send confirmation information to token issuing system to complete token issuing.

Under some special circumstance, Blockchain-based platforms will apply to leaders for issuing and appraisal. For instance, when works can not be evaluated based on current smart contracts, leaders should evaluate them and turn these works into smart contracts to improve current appraisal system. Another circumstance is that leaders offer some irregular works to employees, which require leaders to submit data of works to Blockchain-based platforms at first to help evaluate the works done by employees.

To ensure fairness and efficiency of token issuing, both employees and leaders can make rules and standards of appraisal and incentive based on Pos to formulate DAO, [19] while regulatory authorities are responsible for monitoring the token issuing system. Due to tampering proof, distribution and encryption of Blockchain-based platforms, it would be difficult to influence the operation of token issuing. As a result, it would improve the acceptable level of employees and leaders on appraisal and incentive system as well as the efficiency of corporate governance.

\subsubsection{Token Exchange}

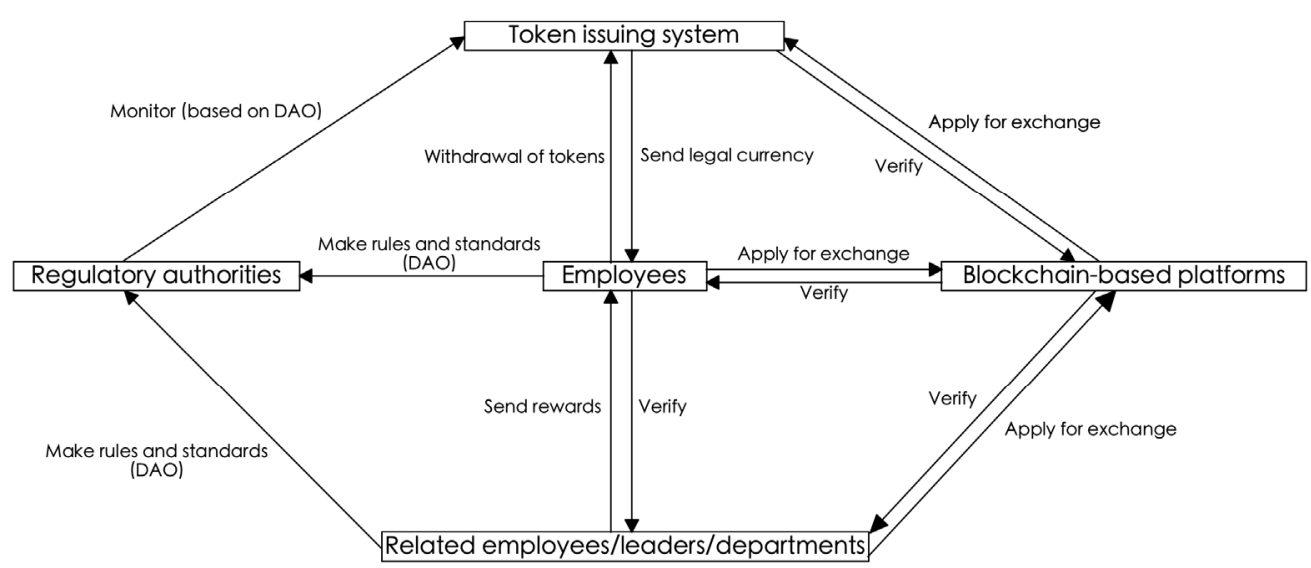

Fig 7. Token exchange

Fig. 7 shows the mechanism of token exchange, which is another important part of the appraisal and incentive system. Token exchange allows employees to exchange token for legal currency or other types of rewards. This will expand the functions of tokens and increase the value of tokens, encouraging employees to work even harder for getting tokens.[20]

When exchanging tokens like Pow with legal currency, employees should apply to Blockchainbased platforms for exchange at first, and Blockchain-based platforms will verify the identities and assets of employees and apply to token issuing system for exchange. Finally, employees will receive legal currency in their digital wallets, and their tokens will be withdrawn by token issuing system.

On the other hand, tokens like Pow can be used to exchange for other types of rewards, which makes it become more attractive and valuable to employees. The steps are like the exchange for legal currency, but Blockchain-based platforms will apply to related employees, leaders or departments for exchange. After receiving rewards, employees should verify and token issuing system will automatically withdraw tokens from digital wallets.

Token exchange has the same mechanism as token issuing in making rules and standards based on Pos, and regulatory authorities will monitor token issuing system to ensure accuracy and fairness. In general, the characteristics of blockchain technology would increase the acceptable level of 
employees and leaders on appraisal and incentive system and the efficiency of corporate governance as well.

\section{Conclusion}

This paper introduces three types of applications in corporate governance that relates to blockchain technology, including operating based on DAO, creating employee performance appraisal and incentive mechanism and cooperating among Multi-departmental employees. As for each application, there are detailed explanations about its mechanism, preconditions and benefits in order to certificate the potential of blockchain technology in the innovation of corporate governance. On the other hand, this paper provides detailed designs of Blockchain-based platforms, operating system and appraisal and incentive system as a guideline for corporations.

Blockchain technology, as a cutting-edged computer technology, shows its superiority in the daily operation of corporations. There are an increasing number of corporations exploring the potentials of blockchain technology in corporate governance since it leads to the amelioration of innovation ability and operation efficiency. Moreover, with trust being build between employees and leaders owing to decentralization, blockchain technology provides new approaches to the increment of corporate competitiveness as well as enterprise digital transformation.

However, corporations that intend to use blockchain technology in their corporate governance still need to face many challenges. This would include the decentralization of corporations, the alternation of leadership, the costs of establishing Blockchain-based platforms etc. Since blockchain technology is still immature, innovation of corporate governance based on blockchain technology is still a problem to be explored.

\section{References}

[1] Bitcoin: A Peer-To-Peer Electronic Cash System, [online] Available: https://bitcoin.org/bitcoin.pdf.

[2] Liu, L. et al., "From Technology to Society: An Overview of Blockchain-based DAO," IEEE Open Journal of the Computer Society, vol. 2, pp. 204-215, 2021.

[3] Junqiao, W. et al., "Management Change in the Blockchain Era", Tsinghua Management Review, no. 10, pp. 70-76, 2018.

[4] Furlonger, D., Uzureau, C., The Real Business of Blockchain: How Leaders Can Create Value in a New Digital Age, 1st ed., Harvard Business Review Press, 2019, pp. 90-99.

[5] Kaal, W. A., "Decentralized Corporate Governance via Blockchain Technology", Annals of Corporate Governance, vol. 5, no. 2, pp. 101-147, 2020.

[6] Diallo, N. et al., "eGov-DAO: a Better Government using Blockchain based Decentralized Autonomous Organization", 5th International Conference on eDemocracy and eGovernment (ICEDEG), pp. 166-171, April 2018.

[7] Xue, L. et al., "Blockchain Technology for Electricity Market in Microgrid", 2nd International Conference on Power and Renewable Energy (ICPRE), pp. 704-708, September 2017.

[8] Wellalage, N.H., Fernandez, V., Thrikawala, S., "Corruption and innovation in private firms: Does gender matter?", International Review of Financial Analysis, vol. 70, pp. 9-11, 2020.

[9] Shin, D.D.H., "Blockchain: The emerging technology of digital trust", Telematics and Informatics, vol. 45, 2019.

[10] Zhong, X., Chuanwei, Z., "What can blockchain do and cannot do?", China Economic Journal, vol. 14, no. 1, pp. 4-25, 2020.

[11] Harbring, C., "On the Effect of Incentive Schemes on Trust and Trustworthiness", Journal of Institutional and Theoretical Economics-Zeitschrift Fur Die Gesamte Staatswissenschaft, vol. 166, no. 4, pp. 690-714, 2010.

[12] Tao, M., Zheng, D., Yuqiao, G., "Enterprise management and innovation driven by blockchain technology", Management Modernization, vol. 39, no. 4, pp. 64-70, 2019. 
[13] Mehra, A. et al., "Distributed leadership in teams: The network of leadership perceptions and team performance", Leadership quarterly, vol. 17, no. 3, pp. 232-245, 2006.

[14] Menghui, L. Et al., "SESCF: A Secure and Efficient Supply Chain Framework via Blockchain-based Smart Contracts", Security and Communication networks, vol. 2021, 2021.

[15] Guangfu, W. et al., "Design and Analysis of Token Model Based on Blockchain Technology", Computer Science, vol. 47, no. 1, pp. 603-608, 2020.

[16] Wei, Z., et al., "Design and analysis of enterprise management system framework based on blockchain", Chinese Journal of Network and Information Security, vol. 5, no. 2, pp. 13-22, 2019.

[17] Yunhua, H., et al., "Research on distributed incentive mechanism based on blockchain", Application Research of Computers, vol. 38, no. 3, pp. 664-670, 2021.

[18] Xiaofeng, M., et al., "Blockchain based supply-chain finance service platform", Big Data Research, vol. 4, no. 1, pp. 13-21, 2018.

[19] Sheng, H., Chunxiao, X., LiangJie, Z., "A Business-Oriented Schema for Blockchain Network Operation",1st International Conference on Blockchain (ICBC), vol. 10974, pp. 277-284, 2018.

[20] Gang, X., Hui, Y., Token Economies, 1st ed., China Business Press, 2020, pp. 139-149.

[21] Akter, S., et al., "Transforming business using digital innovations: the application of AI, blockchain, cloud and data analytics", Annals of Operations Research, 2020. 